\title{
X-ray observations of a large-scale solar coronal shock wave
}

\author{
J. I. Khan ${ }^{1,2}$ and H. Aurass ${ }^{3}$ \\ 1 Mullard Space Science Laboratory, University College London, Holmbury Saint Mary, Dorking, Surrey, \\ RH5 6NT, UK \\ 2 Stationed at: Institute of Space \& Astronautical Science, 3-1-1 Yoshinodai, Sagamihara, Kanagawa 229-8510, \\ Japan \\ 3 Astrophysikalisches Institut Potsdam, An der Sternwarte 16, 14482 Potsdam, Germany
}

Received 14 September 2001 / Accepted 27 November 2001

\begin{abstract}
We report observations of a propagating disturbance in the solar corona observed in emission in soft X-ray images from the Yohkoh Soft X-ray Telescope (SXT). The disturbance was associated with a flare which began at about 09:04 UT on 1997 November 03. This flare was associated with a type II radio burst observed at decimetric-dekametric wavelengths by the Astrophysikalisches Institut Potsdam Radio Spectrograph. H $\alpha$ data from Kanzelhöhe Solar Observatory show that a Moreton wave was associated with this event. Moreover, Solar and Heliospheric Observatory Extreme Ultraviolet Imaging Telescope (EIT) $195 \AA$ data show an 'EIT wave' associated with this event. Extrapolations of the leading edge of the propagating soft X-ray disturbance show a close association with both of these wave features. The soft X-ray disturbance is observed to travel with a speed of about $546 \mathrm{~km} \mathrm{~s}^{-1}$. Using Nançay Radioheliograph data we directly determine the source locations of the type II radio burst. These are found to be located close to the soft X-ray disturbance and show motions consistent with the soft X-ray motions. These results lead us to conclude that the "SXT wave" is the coronal counterpart of a Moreton wave, analogous to EIT waves, i.e., it is the first confirmed direct observation of a solar coronal shock wave in X-rays.
\end{abstract}

Key words. Sun: corona - Sun: flares - Sun: radio radiation - Sun: UV radiation - Sun: X-rays, gamma rays shock waves

\section{Introduction}

Type II solar radio bursts seen in dynamic radio spectrograms (Wild \& McCready 1950; Wild 1950; Wild et al. 1954; Roberts 1959; Wild 1985) were the first indirect observations of large-scale shock waves in the solar corona. It was Uchida (1960) who correctly associated this phenomenon with collisionless fast-mode magnetohydrodynamic shock waves. The passage of a shock wave in the corona is believed to give rise to plasma oscillations which generate (electrostatic) Langmuir waves which are converted into (electromagnetic) type II burst radio emission. For a recent account of observations and theory related to type II solar radio bursts the reader is referred to Aurass (1997) and Mann (1995), respectively.

Another suggested manifestation of large-scale shock waves on the Sun is the "Moreton wave" (also known as flare wave). Moreton waves are arc-like features observed in $\mathrm{H} \alpha(6563 \AA$ ) (and particularly in off-band $\mathrm{H} \alpha$ ) traveling away from an $\mathrm{H} \alpha$ flare site (Moreton 1960, 1961; Moreton \& Ramsey 1960; Ramsey \& Smith 1966). The generally

Send offprint requests to: J. I. Khan,

e-mail: jkhan@spd.aas.org accepted physical description of these propagating disturbances in the chromosphere is in terms of a model invoking coronal shock waves (Meyer 1968; Uchida 1968; Uchida et al. 1973).

Recently, there has been considerable interest in $195 \AA$ images from the Extreme Ultraviolet Imaging Telescope (EIT) (Delaboudinière et al. 1995) on board the Solar and Heliospheric Observatory (SOHO) (Domingo et al. 1995) which have shown wave-like structures in the solar corona (Moses et al. 1997; Thompson et al. 1998, 1999). It has been suggested that these bright propagating EUV features are signatures of the coronal shock waves believed to cause chromospheric Moreton waves. Transient propagating disturbances in the corona have also been observed in 195 and $171 \AA$ images from the Transition Region and Coronal Explorer (TRACE) mission (Wills-Davey \& Thompson 1999).

Delannée \& Aulanier (1999) and Delannée (2000) have suggested that "EIT waves" are not related to shock waves but merely indicate regions of magnetic field compression or electric currents during a magnetic reconnection process involving the opening of magnetic field lines. However 
Delannée (2000) did not discuss observations at wavelengths (such as radio or $\mathrm{H} \alpha$ ), which might indicate associations, or otherwise, with shock waves. Two of the three events examined by Delannée (2000) (one of which is the event examined by Delannée \& Aulanier 1999) have published reported associations with coronal type II bursts, and hence shock waves (Klassen et al. 2000; Leblanc et al. 2000; Solar Geophysical Data, 641(I), 98; 643(I), 123). The third event on 1998 June 13 is also associated with a type II burst as seen in the Astrophysikalisches Institut Potsdam (AIP) Radio Spectrograph (Mann et al. 1992), although this radio event has not been reported previously, nor the spectrogram published. Based on a qualitative examination of a series of events seen from 1997 November 1-6 Delannée et al. (2000a, 2000b) suggested that EIT waves may simply be bright coronal arcs due to the projection (of the low coronal material) of an expanding bubble-like or loop-like coronal mass ejection onto the solar disk. The event discussed in this paper is one of the events examined by Delannée et al. (2000a, 2000b). As will be seen below we find that the EIT wave associated with this event is closely associated with an $\mathrm{H} \alpha$ Moreton wave and a type II burst, suggesting it is related to a shock wave.

Few comparative studies have been carried out examining the relation between EIT waves and radio observations. A comparison of EIT waves with coronal type II bursts seen in spectrograph observations indicates that both may be signatures of the same phenomenon (Klassen et al. 2000). We know of only one published account comparing a type II burst location with an EIT wave (Gopalswamy et al. 2000). Nonetheless, the few results obtained so far do suggest that EIT waves are associated with coronal shock waves. We are aware of three publications comparing EIT waves with Moreton waves (Thompson et al. 2000; Pohjolainen et al. 2001; Warmuth et al. 2001). These found the different wave signatures to be roughly co-spatial, supporting the suggestion that EIT waves may be a coronal counterpart of chromospheric Moreton waves.

Timing arguments and similar values for the derived speeds have been used to support the suggestion of a close relation between Moreton waves and coronal type II bursts (Moreton 1964; Smith 1968; Smith \& Harvey 1971; Wild \& Smerd 1972). While Kai (1969) found type II burst locations apparently lying on an arc centered on a flare and inferred a relation with a shock wave, the only published account of a direct comparison of type II burst locations with $\mathrm{H} \alpha$ Moreton waves is that of Harvey et al. (1974).

To date, there have been only speculative suggestions that some soft X-ray features observed in the solar corona may have been observations of large-scale shock waves. These were based on the behaviour of the soft X-ray features and the apparent overlap in time with metric type II bursts seen in radio spectrograms (Hudson \& Karlický 2000; Khan \& Hudson 2000).
In this paper we report observations of a transient, large-scale, propagating feature seen in soft X-ray images associated with a major solar flare on 1997 November 3. This event is associated with an $\mathrm{H} \alpha$ Moreton wave, an EIT wave, and a coronal type II burst. We examine the nature of the soft X-ray disturbance and compare with radio spectrograph data as well as $\mathrm{H} \alpha$, EIT $195 \AA$ and radio images. Our results suggest that the soft X-ray disturbance observed in the early stages is the first clearly demonstrated signature of a large-scale coronal shock wave observed in soft X-rays.

\section{Observations}

\subsection{Principal data sources}

The principal data presented in this paper are soft X-ray images from the Soft X-ray Telescope (SXT) (Tsuneta et al. 1991) on board the Yohkoh satellite (Ogawara et al. 1991). The SXT is sensitive to soft X-ray photons in the energy range $\sim 0.28-4 \mathrm{keV}$ corresponding to wavelengths of $\sim 3-45 \AA$. It images solar coronal plasma hotter than about $2 \mathrm{MK}$. Using standard software we corrected the SXT data to subtract the dark current and stray light, and align the images to each other. The resulting linear pixel values were then scaled using the compression formula given in Tsuneta et al. (1991). This scaling exaggerates the apparent brightness of the faint features making them easier to see. For SXT difference images we take the differences of the raw (unscaled data) and then truncate the values to the range necessary to show the features of interest. The SXT images and difference images are displayed using a reverse linear black and white color table (where darker denotes higher soft X-ray intensity). These measures are helpful because of the large dynamic range of the soft X-ray emission observed.

In this paper we are interested in comparing the SXT data with imaging data which have previously shown wave-like features associated with large-scale shock waves. Of the four filter images taken by the SOHO EIT the ones centered on the Fe XII line at $195 \AA$ have been found to be the most effective in finding so-called "EIT waves". We present EIT $195 \AA$ images which show a wave-like disturbance for this event. The EIT $195 \AA$ images generally show plasmas in a narrow temperature range which peaks around $\sim 1.6 \mathrm{MK}$ (Delaboudinière et al. 1995). We also present $\mathrm{H} \alpha(6563 \AA)$ images from Kanzelhöhe Solar Observatory, Austria which show a chromospheric Moreton wave. The $\mathrm{H} \alpha$ images from Kanzelhöhe Solar Observatory are cinematographic images taken with a time cadence of about $4 \mathrm{~min}$.

Radio data from the Astrophysikalisches Institut Potsdam (AIP) Radio Spectrograph (Mann et al. 1992) are used to identify the radio bursts associated with this event; specifically, the type II radio burst. The AIP spectrograph is a set of four sweep spectrometers which span the frequency range $(40-800 \mathrm{MHz})$, covering dekametric to decimetric wavelengths with a sampling rate of $0.1 \mathrm{~s}$. 


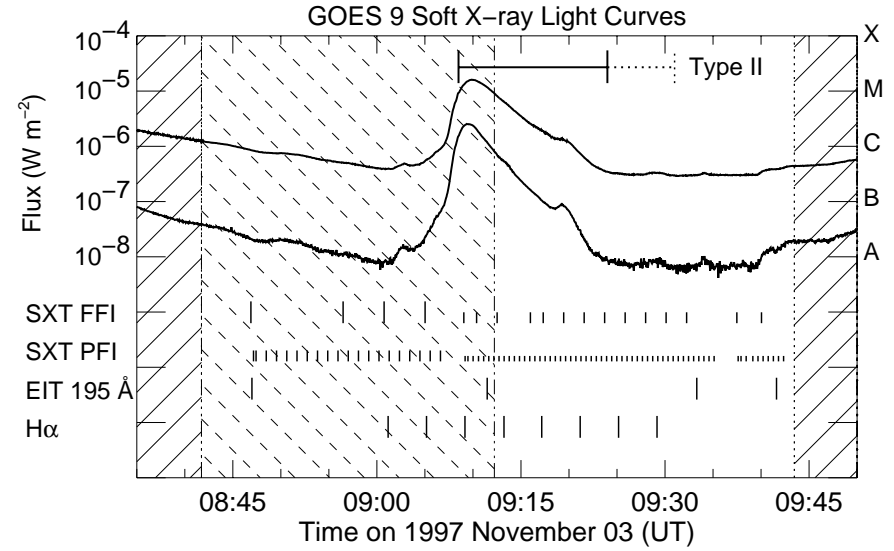

Fig. 1. The soft X-ray flux for the low energy channel $(\sim 1-$ $8 \AA$, the higher flux $)$ and for the high energy channel $\left(\sim 0.5^{-}\right.$ $4 \AA$, the lower flux) of the GOES 9 satellite. This shows the flare associated with the SXT propagating disturbance and the temporal coverage of some of the data examined in this paper.

Type II bursts are most commonly seen starting at or below $150 \mathrm{MHz}$ in the fundamental mode of the plasma frequency (Nelson \& Melrose 1985). For this event the second harmonic mode of the type II radio burst was seen starting in the decimetric range. This is fortunate because it enables us to directly image the source locations of the type II radio burst using data obtained from the Nançay Radioheliograph (NRH) (Kerdraon \& Delouis 1997). For this event, images of the Sun at the five NRH frequencies of 164.0, 236.6, 327.0, 410.5 and $432.0 \mathrm{MHz}$ are available and are useful in the analysis of the type II radio burst. To identify and interpret the type of radio feature being imaged at a given time by the NRH it is necessary to compare closely with the radio spectrograph data. To assist with this comparison we compute the whole-Sun fluxes for each of the NRH frequencies. This is done using uncleaned NRH data (the cleaning routine does not alter the whole-Sun flux density). For analysis of the NRH images we use data cleaned using the standard NRH software.

\subsection{Context observations}

Before examining in detail the Yohkoh SXT data for the propagating soft X-ray disturbance we briefly describe the context in which this event occurred.

The disturbance seen in the SXT images was observed moving to the northeast away from, roughly speaking, the vicinity of a major flare in the northern part of the active region associated with NOAA sunspot region 8100. This flare, of X-ray class M1.4 and optical class 1B, began at about 09:04 UT on 1997 November 3. The heliographic coordinates of the center of the soft X-ray flare were roughly S17W18.

In Fig. 1 we present GOES soft X-ray fluxes to illustrate the soft X-ray flare associated with this event and as a time-line to discuss the temporal coverage of the various data sources used in this paper. The fluxes are shown as thick solid curves. Comparison with SXT data show that the small bump from about 09:01:40-09:03:50 UT did not originate from the loops which later flared. Soft X-ray brightening of the flare region was associated with the initial rise in the GOES flux from about 09:04-09:07 UT, as well as with the steeper rise from about 09:07 UT to the soft X-ray peak (which was at about 09:09:50 UT and 09:09:20 UT for the low and high channels, respectively). Superimposed on this plot are hatched areas representing intervals of Yohkoh satellite night (solid hatches) and when Yohkoh was in the South Atlantic Anomaly (SAA) region of the inner van Allen radiation belts (dashed hatches).

Also superimposed in Fig. 1 are small vertical tick marks indicating the times of some of the data examined in this paper. Outside the plot axes, to the left of these bands of ticks, labels indicate the types of images these ticks correspond to. The times of all of the EIT $195 \AA$ images for the interval of the plot are shown. We also show the times of $\mathrm{H} \alpha$ images from Kanzelhöhe Solar Observatory, for the interval 09:01:10-09:29:10 UT. The AIP Radio Spectrograph data and the NRH radio image data started well before the flare and continued until well after the event. These data sets observed the event from beginning to end with high time resolution. Consequently, we do not explicitly indicate the times of those data sets in Fig. 1. However we do show the interval of the type II radio burst determined from the AIP spectrograph data (solid line). Published reports in Solar Geophysical Data indicate that this type II radio burst was observed at frequencies below the lowest AIP spectrograph frequency. We indicate the interval of the reported type II burst extension from $35-40 \mathrm{MHz}$ with a dashed line in Fig. 1.

In general, Yohkoh SXT observations consist of fullframe images (FFIs) of the whole Sun, as well as high time cadence images of selected regions, referred to as partial-frame images (PFIs). For times of the SXT images indicated in Fig. 1, the size of the ticks is indicative of the spatial resolution of the pixels. For the FFIs the larger ticks indicate $256 \times 256$ pixel images of quarter resolution (9.82 arcsec) pixels, while the smaller ticks indicate $512 \times 512$ pixel images of half resolution $(4.91 \mathrm{arcsec})$ pixels. For the PFIs the smallest ticks indicate $128 \times 128$ pixel images of full resolution $(2.45$ arcsec $)$ pixels, while the larger ticks indicate $64 \times 64$ pixel images of half resolution pixels. For this particular event, the fields of view of both types of PFIs are the same.

\subsection{Yohkoh soft $X$-ray images}

When a flare occurs which is sufficiently intense, Yohkoh normally switches into flare-mode, in which high time cadence partial-frame images (PFIs) of the flaring region are taken, but no FFIs are taken. However when Yohkoh passes through the SAA region, high voltages on board are reduced and flare-mode is not triggered. Thus only SXT FFIs and quiet-mode PFIs are taken, even if a large flare occurs. This was the situation for the event examined in 
Yohkoh SXT 1997 November 03 09:12:30.9 UT

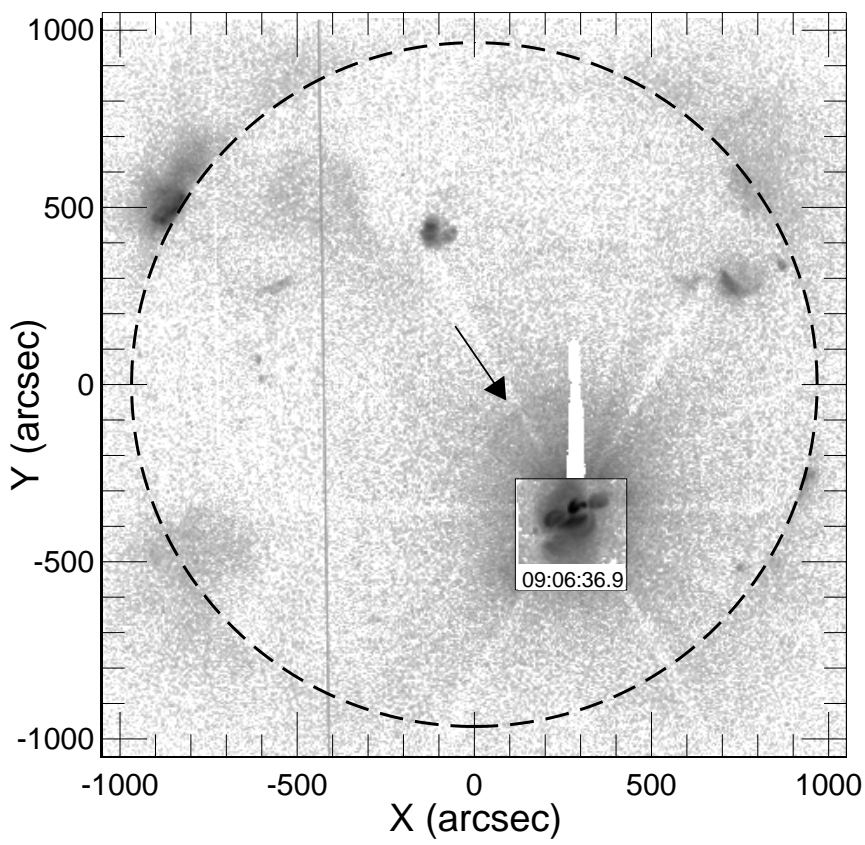

Fig. 2. A Yohkoh SXT whole-Sun image taken at 09:12:30.9 UT on 1997 November 3 showing the propagating soft X-ray disturbance emanating from active region 8100. Superposed on this image is a partial-frame image of the flaring active region at 09:06:36.9 UT on the same day, near the start of the flare.

this paper. One fortunate consequence is that whole Sun images were taken during this event, which is not normally the case for Yohkoh observations of flares. Thus a wider field of view was observed than is usually the case for large flares observed by Yohkoh.

In Fig. 2 we present an example of an SXT FFI showing the propagating disturbance (indicated by an arrow). Some regions of the CCD were saturated during the flare. The flare not only saturated the CCD in the immediate vicinity of the flaring region but also caused charge to spill into adjacent pixels causing them to saturate. This results in a long vertical strip of saturated pixels north of the flaring region. All saturated pixels were set to zero and appear white in Fig. 2. The high intensity of soft X-rays entering the SXT (due to the flare) also results in other instrumental artifacts in the data. Specifically, a region of enhanced emission around the flaring region due to the scattering of $\mathrm{X}$-ray photons (e.g., compare Figs. $3 \mathrm{a}-\mathrm{c}$ which will be discussed shortly), as well as two types of patterns of voids in soft X-ray emission: (i) a star-burst-like pattern extending approximately radially away from the flare region, and (ii) thin concentric arcs approximately centered on the flare region. The first type of void pattern is seen in Fig. 2, while both types of void patterns are seen clearly in some of the figures which will be presented later (e.g., Figs. 3b,c).

To show the structure of the active region we have superposed a half resolution PFI near the start of the soft $\mathrm{X}$-ray flare on top of the FFI in Fig. 2. The edge of this PFI shows the field of view of all the PFIs taken during this event. We have examined the half and quarter resolution PFIs but we do not find any evidence for a propagating disturbance in those images. The exposure durations for the PFIs may not have been long enough to show such a faint feature. Another possibility is that the propagating feature first appears in soft X-rays outside the field of view of the PFIs, i.e., further away from the flaring region.

Portions of all of the FFIs showing the propagating feature are presented in Fig. 3. Note that this set consists of images taken with different exposure durations and different filters. The images were scaled to span the full range of display values for each individual image. Thus the relative differences in brightness between the images cannot be compared directly from this figure. However displaying the images in this way shows the most important features at the time of each image and this helps to show the fainter features in some of the images.

In Fig. 3a we show an image taken before the flare associated with the propagating disturbance. (In order to show an image without flaring in the FFI we had to go to this much earlier image). Figure $3 \mathrm{~b}$ shows an image a few minutes after the start of the soft X-ray flare. A propagating feature is not yet observed. Figures $3 \mathrm{~b}-\mathrm{g}$ are sequential without the omission of any SXT FFIs. In the next image, Fig. 3c, a transient propagating feature with a sharply defined leading edge is apparent. A clear leading edge is also seen for the disturbance in Fig. 3d. These leading edges are indicated with arrows in Figs. 3c and 3d. A marginally visible feature which may be related to the earlier propagating features is indicated with an arrow in Fig. 3e. In Fig. 3f we see a propagating feature with a relatively sharply defined feature near the trailing edge of the disturbance. At the time of these last two images the leading edge is too diffuse to discern clearly. An edge of the disturbance is indicated by an arrow in Fig. $3 \mathrm{~g}$. It is not clear whether this is related to the leading edges seen earlier or the possible trailing edge seen in Fig. 3f. We do not see a propagating disturbance in subsequent images. The third FFI after (g) is shown in (h), because the two earlier images are taken with a low exposure duration and are too faint too discern any features outside of the active region. Despite the less than optimal observations using a variety of filters and exposure durations, a propagating feature can be seen in the raw, corrected Yohkoh SXT data.

To show the propagating feature more clearly we take differences of the images in Figs. 3c-h. We also zoom the images. These difference images are presented in Fig. 4. They show differences of exposure normalized images for the times indicated. The difference images are displayed over a narrow range of difference values (which varies for each image) necessary to show the propagating feature in each image clearly. As in Fig. 3 the relative differences in brightness between the images do not reflect actual differences in intensity between the images. In this figure we take difference images using the same filter in the 

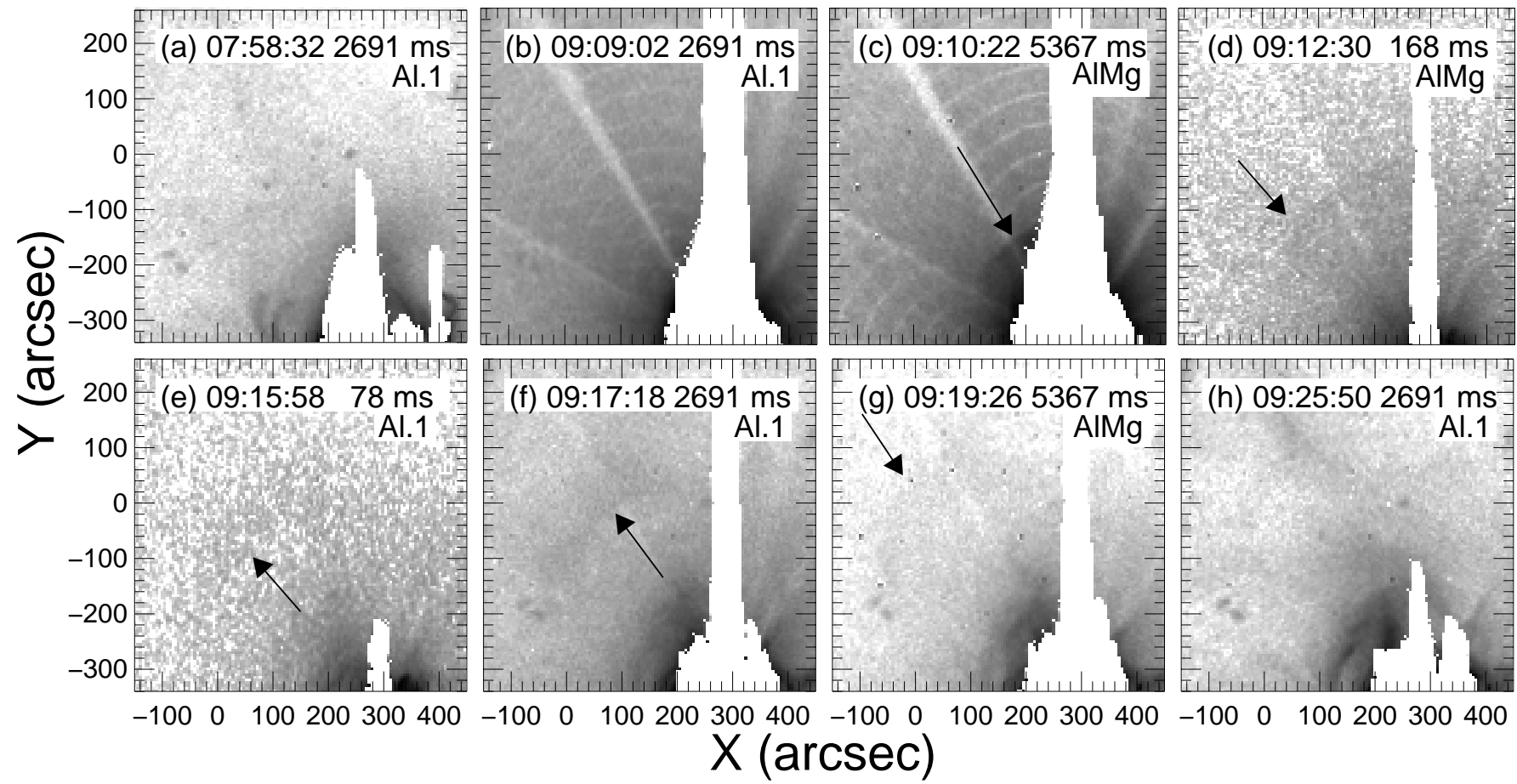

Fig. 3. A sequence of portions of SXT whole-Sun images showing a propagating soft X-ray disturbance. The image times, the original exposure durations, and the soft X-ray filters of the images are indicated at the top of each of the panels.


Fig. 4. Portions of SXT whole-Sun difference images scaled to show the propagating soft X-ray disturbance. The times of the images (in UT) used to construct each difference image are shown at the top of each panel. 

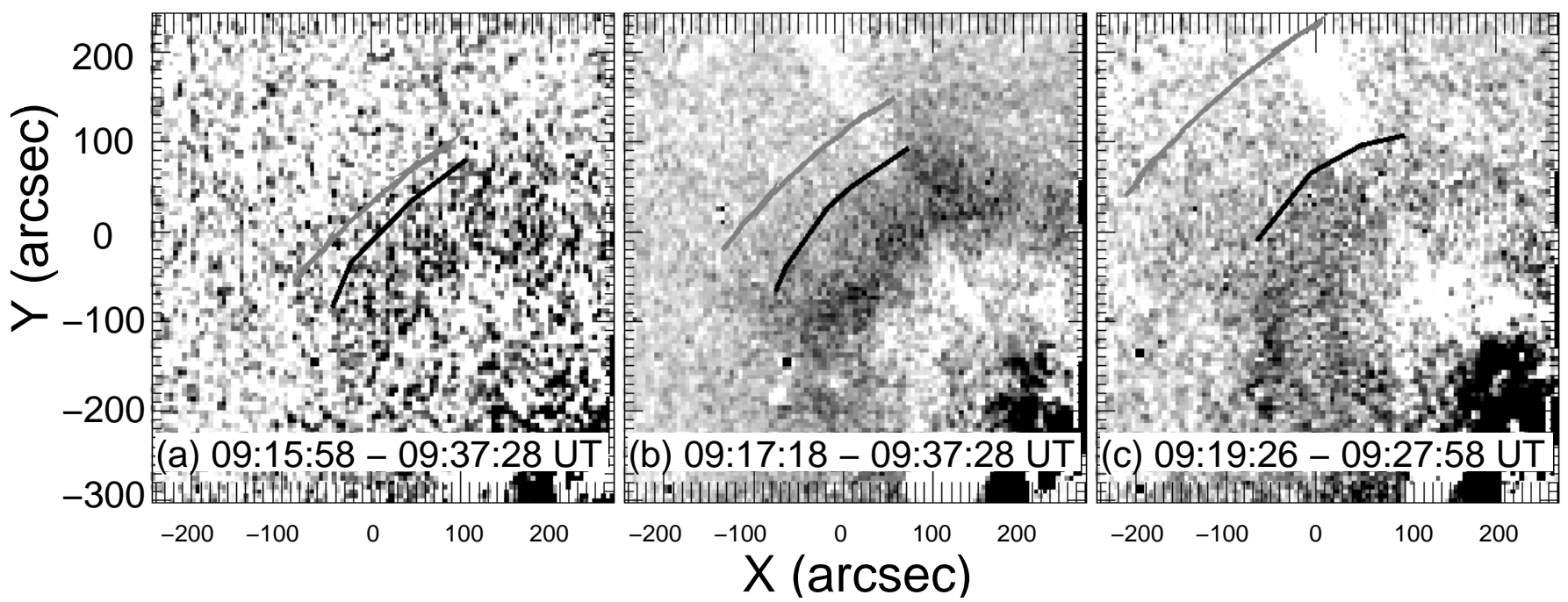

Fig. 5. Portions of SXT difference images similar to Figs. 4c-e. In each panel we have drawn the estimated location of the leading edge (black lines) and the extrapolated location of the leading edges seen in Figs. 4a,b (gray lines).

subtrahend as the minuend. We indicate the apparently propagating features with arrows in Figs. $4 \mathrm{a}-\mathrm{e}$.

We now indicate briefly some noteworthy morphological features of the images shown in Fig. 4. In Figs. 4a,b a clear leading edge is seen but a distinct 'trailing' edge is not seen. Behind these leading edges is a region of emission of similar intensity level to the leading edges. Behind that is the region of saturated pixels. However Figs. 4c-e do show a disturbance of higher intensity than regions both upstream and downstream of it. Furthermore, in Fig. 4e the soft X-ray feature appears to consist of perhaps two roughly concentric disturbances (indicated by white arrows).

Although the disturbance is seen in Figs. 4c-e, it is somewhat problematic identifying with a reasonable degree of confidence well-defined features in those images which may be associated with each other. However for Figs. 4a,b it appears reasonable to suppose that the two leading edges may be related to each other.

We calculated the average speed between the leading edges seen in the images shown in Figs. 4a,b by the following method. First we visually selected points along each leading edge by "pointing and clicking". Then we spline interpolated these points to generate a larger number of points along each edge. Next we determined the closest spline-interpolated point lying along the edge of the feature in Fig. 4b for each spline-interpolated point lying along the edge of the feature in Fig. 4a. Thus for each point on the leading edge of Fig. 4a we have a corresponding point on the leading edge of Fig. 4b. Measuring the distance between each pair of points in units of SXT pixels and knowing the size scale of the SXT pixels we determined the implied speed of propagation for each of the points along the leading edge of Fig. 4a to the the corresponding points along the leading edge of Fig. 4b. From these values we estimate the average speed between the two leading edges to be $546 \pm 21 \mathrm{~km} \mathrm{~s}^{-1}$, where the error indicates the standard deviation of the speeds combined in quadrature with an estimate of the uncertainty of the location of the edges. This procedure of effectively drawing the shortest line segments between the leading edges is nothing more than a ray-tracing technique based on Huygens' principle to predict the propagation of a wavefront. Recently, Wills-Davey \& Thompson (1999) used a similar technique to examine a propagating disturbance seen in TRACE data.

By fitting a straight line through the pairs of matching points along the leading edges of Figs. $4 \mathrm{a}$ and $4 \mathrm{~b}$ we can extrapolate the position of the SXT leading edge to other times. This assumes the SXT leading edge traveled with a constant speed.

In Fig. 5 we present portions of the full-disk images corresponding to those shown in Figs. 4c-e, but with a wider field of view. In each panel we have drawn (with solid gray lines) the position of the extrapolated leading edge (seen in Figs. $4 \mathrm{a}$ and 4b, i.e., assuming $546 \mathrm{~km} \mathrm{~s}^{-1}$ ) to the relevant times in each panel. Although we are not able to determine with a reasonable degree of confidence the leading edges of the disturbances seen in Figs. 4c-e; nevertheless, we draw our best estimates of where the leading edge may be located in Fig. 5 (solid black lines). The advance of the emission features between Figs. 5b,c appears to indicate possible motion of the disturbance. However, we do not find significant displacement of the estimates of the leading edges of the features seen in Figs. 5a-c. We also note that the ridge seen in emission behind our estimate of the leading edge in Fig. 5b may give rise to the emission features seen in Fig. 5c (one of which is close to our estimate of the leading edge). If so, then this indicates that our estimates of the leading edges are not tracking the same feature in Figs. 5b,c. The difference in morphology and propagation characteristics of the features seen in Figs. 4c-e compared to those seen in Figs. 4a,b suggests they may not be closely related to the leading edge 


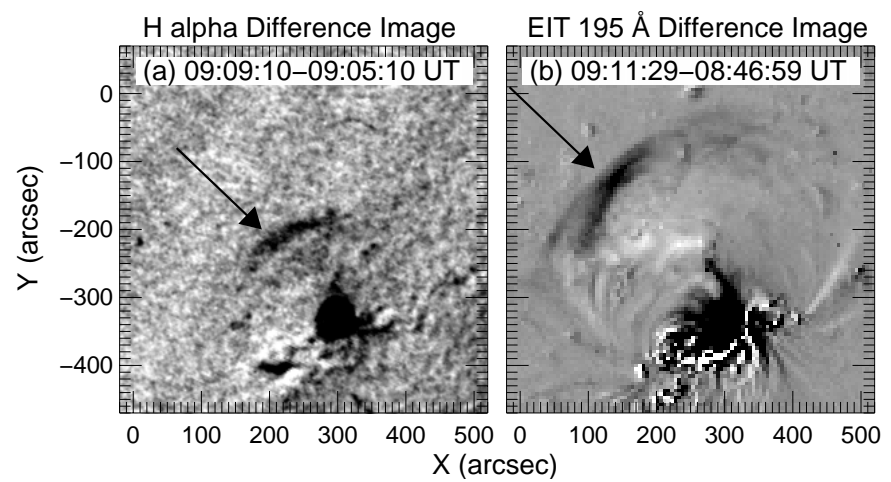

Fig. 6. An $\mathrm{H} \alpha$ difference image (raw data courtesy W. Otruba and A. Warmuth) showing a Moreton wave and an EIT $195 \AA$ difference image (raw data courtesy the SOHO-EIT Consortium) showing a possible EIT wave. These difference images are displayed using a reverse linear black and white color table (where darker denotes higher intensity).

of the shock wave. Instead they may indicate ejecta which could have been the driver of the shock wave which later decelerated, or they may indicate shocked coronal plasma left behind after the passage of the shock wave. Thus the marked separation of the extrapolated and observed leading edges in Figs. 5b,c probably does not indicate deceleration of the shock wave, as might have been concluded otherwise. We conclude that only Figs. 4a,b are useful as observations of the shock wave.

\section{4. $\mathrm{H} \alpha$ and SOHO EIT $195 \AA$ images}

$\mathrm{H} \alpha$ data from Kanzelhöhe Solar Observatory show a clear Moreton wave in an image taken at 09:09:10 UT. To show this feature we present a portion of a difference image of that whole-Sun $\mathrm{H} \alpha$ image with the earlier image taken at 09:05:10 UT in Fig. 6a. We do not see a clear Moreton wave in the image at 09:13:14 UT or in subsequent images. The EIT $195 \AA$ data for this event were briefly discussed in Klassen et al. (2000) and Delannée et al. (2000b). SOHO EIT $195 \AA$ data show an apparent EIT wave in the image taken at 09:11:29 UT. In Fig. 6b we show this feature with a portion of a difference image of that image with the previous EIT $195 \AA$ image taken at 08:46:59 UT. The range of difference values in both Figs. $6 \mathrm{a}$ and $6 \mathrm{~b}$ are truncated to the range necessary to show the wave features in each case.

The Moreton wave was observed $72 \mathrm{~s}$ prior to the first observation of the SXT propagating disturbance. The EIT $195 \AA$ wave was observed between the times of the two SXT images showing a clear leading edge (see Figs. 4a and $4 \mathrm{~b}$ ). Using the same technique that was used for Fig. 5 we compare the actual locations of the Moreton wave and the EIT $195 \AA$ wave with positions of the SXT leading edge extrapolated (using $546 \mathrm{~km} \mathrm{~s}^{-1}$ ) to the times of those features.

In Fig. 7 we show a zoomed portion of the difference image shown in Fig. 4a with a superposed raw (not

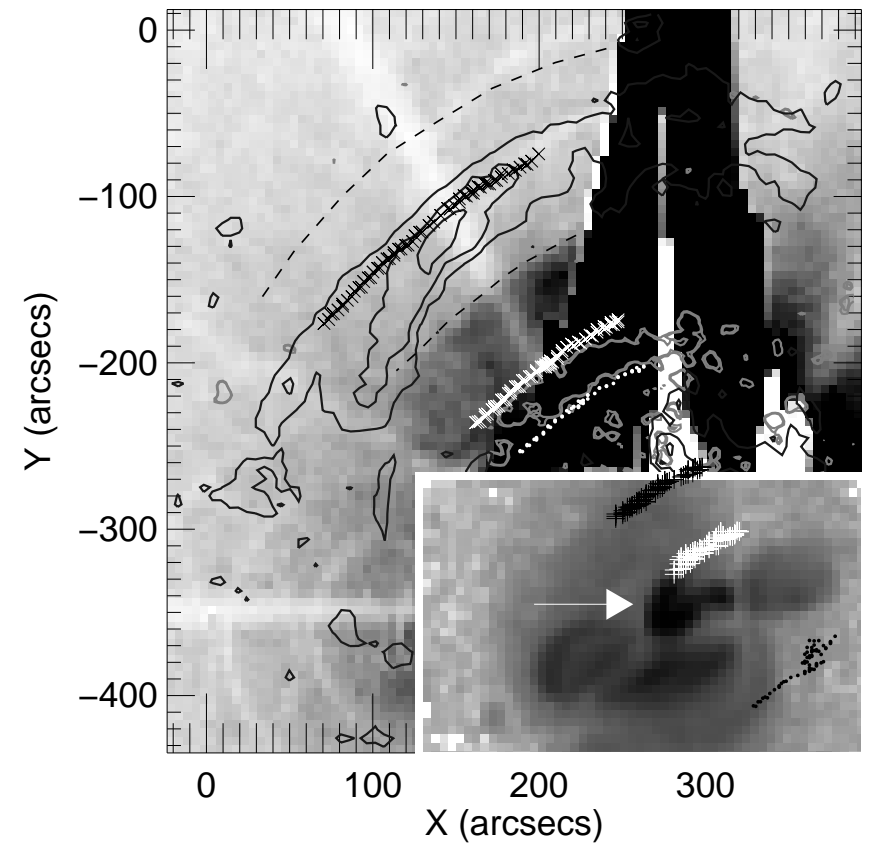

Fig. 7. This figure shows the difference image shown in Fig. 4a with a superposed portion of a raw PFI taken at 08:52:44 UT (to show the active region and flaring structures) with lines and symbols indicating various features and extrapolated locations as discussed in the main text.

difference) half resolution PFI (scaled as other raw images shown in this paper) taken at 08:52:44 UT to show the flaring part of the active region. We have indicated the locations of the leading edges of the SXT features for Figs. 4a and 4b with dashed black lines. We have also overplotted contours for the EIT $195 \AA$ wave (solid black lines) and the $\mathrm{H} \alpha$ Moreton wave (solid gray lines). The extrapolated positions for the time of the EIT $195 \AA$ wave are shown with black $\times$ symbols, while those for the Moreton wave are shown with white $\times$ symbols. This figure shows that the extrapolated leading edge of the SXT propagating disturbance seen in Figs. 4a,b matches closely both the Moreton wave and EIT $195 \AA$ wave locations. This suggests that the chromospheric Moreton wave, the EIT coronal wave, and the SXT feature may all be closely related to each other.

We also extrapolated the SXT leading edge to the start time of the type II radio burst as seen in the AIP spectrogram. The type II radio burst was first clearly seen at 09:08:30 UT as second harmonic mode emission. The extrapolated front at this time is shown with white dots in Fig. 7. Assuming this extrapolated location is a reasonable indication of the actual starting position of the type II burst, then this indicates the type II burst first appeared about $85 \mathrm{Mm}$ from the edge of the flaring kernel. This is similar to the result of Klein et al. (1999) who found that the type II burst first appeared about $100 \mathrm{Mm}$ from the flaring kernel for the event they examined. Figure 7 shows the northern and eastern edges of the field of view of the PFI region. Thus the type II burst may have first appeared outside the PFI field of view. 
We extrapolated the SXT leading edge to the start time of the flare as indicated by the start of the initial rise in the GOES flux at 09:04 UT (shown with black dots in Fig. 7) and to the start time of the steeper rise in the GOES flux at 09:07 UT (shown with black + symbols in Fig. 7). The region which flares is shown with a white arrow in Fig. 7 (the soft X-ray emitting region which appears dark). Extrapolating the SXT leading edge to the edge of this soft X-ray feature (as indicated by the white + symbols in Fig. 7) we find that this location implies a time of approximately 09:06 UT.

Two qualitatively different models have been proposed to produce the shock waves inferred from observations of coronal type II bursts. One is a piston-driven scenario where mass motion, either associated with the flare energy release process or a coronal mass ejection (as seen in coronagraphs) directly drives a shock wave ahead of the moving material (Maxwell \& Dryer 1982; Maxwell et al. 1985). The other scenario is a freely propagating blast wave initiated by a pressure pulse (Vršnak \& Lulić 2000). Incidentally, it should be noted that in the first scenario once the piston stops, the previously directly driven shock wave may then freely propagate as a blast wave (Nelson \& Melrose 1985). Concerning the pressure pulse mechanism, Vršnak \& Lulić (2000) suggested two possible scenarios for the explosive expansion of a flaring volume to produce shock waves. One is in situ heating at the presumed energy release (magnetic reconnection) site above the tops of soft X-ray loops, the other is chromospheric evaporation along the legs of the flaring loops.

For the event examined here the launch time of the wave might have been either the relatively slower initial rise in the GOES flux from 09:04 UT or the steeper rise in the GOES flux from 09:07 UT. Neither of these times is viable for the proposed magnetic reconnection scenario for large-scale shock waves since the launch site is required to be above the soft X-ray loop-top region near 09:06 UT, assuming this extrapolated start time is reasonable. However, the second suggestion associating largescale shock waves with chromospheric evaporation may be a possibility. Since the half-length of the flaring structure is about $20 \mathrm{Mm}$, for plasma which starts evaporating from 09:04 UT to reach the loop-top region by the inferred start time of 09:06 UT at the flare site requires a flow speed of about $160 \mathrm{~km} \mathrm{~s}^{-1}$, which is close to the blue-shifts measured in flares using soft X-ray spectrometers, such as the Yohkoh BCS (e.g., Mariska 1994; Ding et al. 1996). Thus the propagating soft X-ray disturbance may be related to chromospheric evaporation if it is associated with the relatively slower rise in the GOES flux from 09:04 UT. However, it cannot be associated with such a source due to the steeper rise in the GOES flux from 09:07 UT since this would imply a start time after the inferred start time of 09:06 UT at the flare site, assuming the inferred speeds are appropriate.

A further possibility is that magnetic reconnection accompanying the steep rise in the GOES flux from 09:07 UT may give rise to rapid restructuring of the mag-

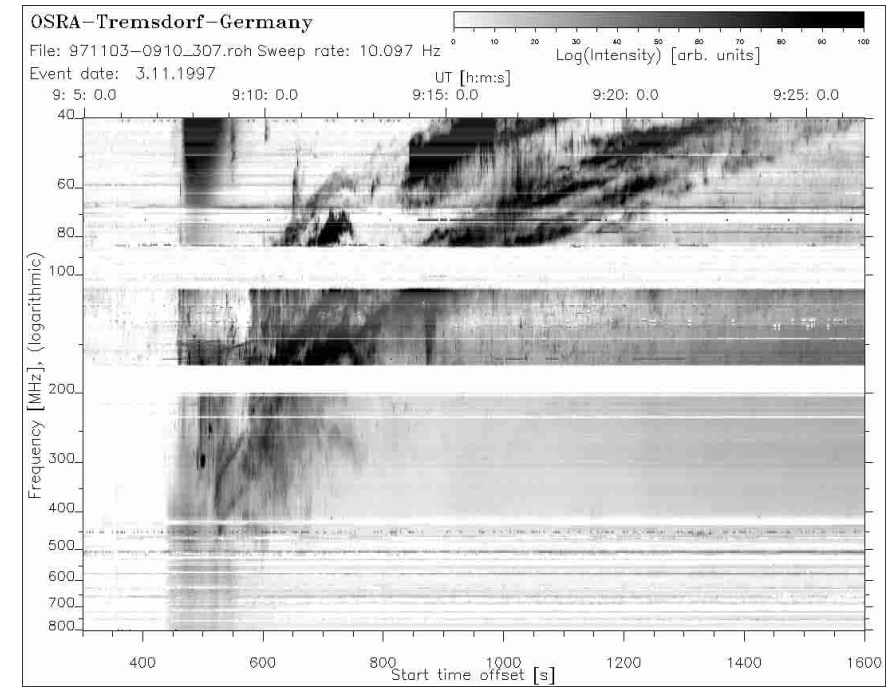

Fig. 8. A spectrogram from the Astrophysikalisches Institut Potsdam Radio Spectrograph showing an overview of the radio bursts associated with the event on 1997 November 3 discussed in this paper.

netic field and accompanying mass ejection of large-scale structures surrounding the flaring region. This may provide the sudden impulse required to launch the (shock) wave from a coronal source remote the flare kernel, e.g., at the 09:07 UT position indicated by black + symbols in Fig. 7. Note we find that the extrapolated position of the leading edge of the propagating SXT disturbance does not converge on the flaring soft X-ray kernel but is offset from that region (compare the white + symbols with the flaring region indicated by the arrow). This may indicate bending of the soft X-ray disturbance as it propagated away from the flaring region, as has been observed with TRACE $195 \AA$ waves (Wills-Davey \& Thompson 1999) and $\mathrm{H} \alpha$ Moreton waves (as shown and explained by Uchida 1970, 1974). Alternatively, this result may provide further support for the suggestion that the soft X-ray disturbance did not originate from the flaring kernel but was associated with (offset) erupting structures further away (to the northeast), similar to the findings of Klassen et al. (1999) and Klein et al. (1999).

\subsection{Radio spectrograph observations}

In Fig. 8 we present a dynamic spectrogram from the AIP Radio Spectrograph which gives an overview of the radio bursts associated with the event examined in this paper. The radio data for this event were briefly discussed in the paper by Klassen et al. (2000) which cataloged EIT wave events associated with type II bursts observed by the AIP Radio Spectrograph in 1997. This event was also one of several reported by Klassen et al. (2001) which show interesting fine structures in the radio spectrograms.

From about 09:07:30-09:09 UT a group of type III radio bursts was seen in the $40-80 \mathrm{MHz}$ range. A strong type II radio burst started at higher frequencies and was 
the dominant radio feature together with some continuum emission in the frequency range 40-750 MHz. The type II burst clearly showed second harmonic component emission extending roughly between 09:08:30-09:27 UT. Each mode was split into at least two bands. (Henceforth we use lane to refer to each collective harmonic component and band to refer to the individual split structures). Two split bands were seen in the early stages, while more than two split bands were seen in later stages. The second harmonic lane was first seen 09:08:30 UT at $\sim 420 \mathrm{MHz}$. The fundamental mode lane is less continuous than the second harmonic one: between 09:12-09:13:30 UT there is an absence of fundamental mode lane emission. The frequency drift rate of the fundamental lane differs before and after the "interruption". From 09:10-09:12 UT it was $\sim-0.50 \mathrm{MHz} \mathrm{s}^{-1}$, while from 09:13:30-09:16:30 UT it was $\sim-0.12 \mathrm{MHz} \mathrm{s}^{-1}$. The change in frequency drift rate might be attributed to the shock wave decelerating, propagating in a direction more orthogonal to the density gradient, or entering a region with a density gradient lower than that assumed in the density model (Karlický et al. 1982; Robinson 1985). Using a density model of the solar atmosphere we can estimate the approximate speed (along the direction of the density gradient) of the shock (presumed to cause the type II radio burst). For the interval 09:10-09:12 UT, the Mann et al. (1999) model implies a speed of $\sim 560 \mathrm{~km}^{-1}$, while the Newkirk (1961) model implies $\sim 1235 \mathrm{~km}^{-1}$, and a fourfold Newkirk model implies $\sim 1893 \mathrm{~km}^{-1}$. For the interval 09:13:30-09:16:30 UT, the Mann et al. model implies $\sim 275 \mathrm{~km}^{-1}$, while the Newkirk model and fourfold Newkirk model imply $\sim 726 \mathrm{~km}^{-1}$ and $\sim 1182 \mathrm{~km}^{-1}$, respectively. Ignoring questions about the directions of propagation, the speed estimated using the Mann et al. (1999) density model gives a value close to the estimated average speed of propagation (of $546 \pm 21 \mathrm{~km} \mathrm{~s}^{-1}$ ) between the leading edges seen in the SXT images of 09:10:22 UT and 09:12:30 UT.

The speed estimated from radio spectrograms is along the direction of the density gradient in the solar atmosphere. If we assume, for the moment a plane-parallel density model for the solar atmosphere and a fairly isotropically propagating shock wave, then we can understand the similarity in the radio spectrogram-derived speed and SXT-derived speed (assuming the SXT leading edge is closely related to the front of the shock wave). If the shock wave propagates isotropically then the horizontal (to the solar photospheric layer) and the vertical speeds would be similar. With the above assumptions, the radio spectrogram-derived speed would be in the vertical direction and the SXT-derived speed would be in the horizontal direction, and would thus be similar.

The second harmonic mode of the type II burst extended from $\sim 450-40 \mathrm{MHz}$. In Fig. 9 we present the spectrogram shown in Fig. 8 but now only showing the frequency range and time interval appropriate for the type II burst at NRH frequencies.

The appropriate intervals for images of the type II burst for each of the NRH frequencies can be determined

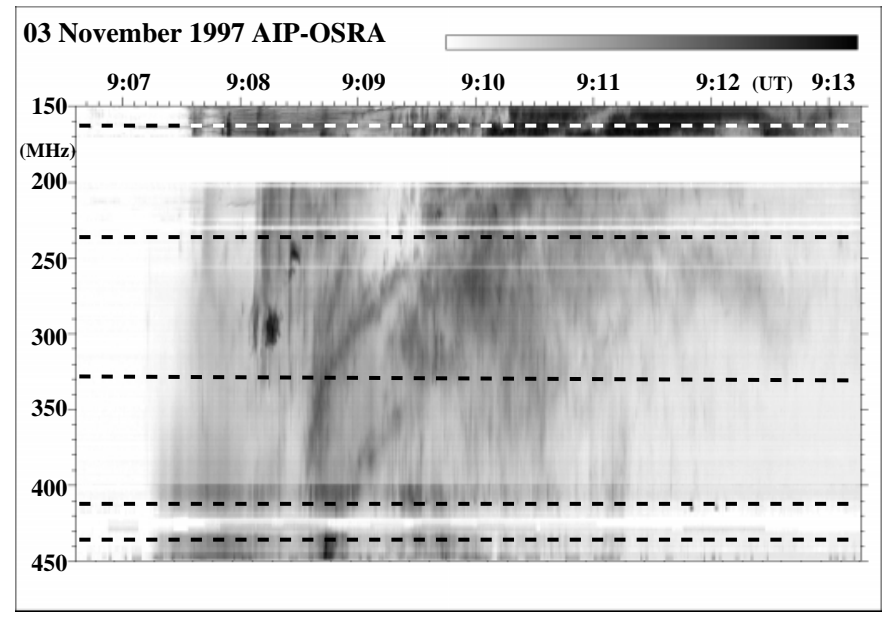

Fig. 9. A spectrogram from the Astrophysikalisches Institut Potsdam Radio Spectrograph showing the type II radio burst over the range of NRH frequencies. The dashed horizontal lines indicate the NRH frequencies of 164.0, 236.6, 327.0, 410.5 MHz, and $432.0 \mathrm{MHz}$. The color bar illustrates the distribution of the logarithmic flux density in arbitrary units.

by examining the spectrogram and comparing plots of the radio fluxes from the AIP spectral data near the $\mathrm{NRH}$ frequencies with the NRH whole-Sun fluxes. The second harmonic mode of the type II burst is clearly observed at all of the NRH frequencies. It appears that fundamental mode emission of the type II burst was also observed at some of the NRH frequencies. We find (not entirely unexpected) complexity in the relation between the fundamental and second harmonic mode sources of the type II burst. Consequently, for simplicity, we discuss only the stronger second harmonic mode sources as reliable indicators of the shock location in this paper.

\subsection{Nançay radioheliograph data}

\subsubsection{Fluxes of the Nançay radioheliograph data}

Before considering the radio images, we first present the whole-Sun fluxes observed at each of the NRH frequencies in Fig. 10. In each panel, corresponding to each frequency observed by the NRH, we have indicated the approximate interval of the second harmonic mode of the type II burst. These temporal limits of the type II burst were determined by examining zoomed portions of the AIP radio spectrograms and comparing the NRH fluxes with the fluxes at similar frequencies in the AIP Radio Spectrograph data. For the second harmonic mode emission we indicate the lower frequency ("outer") split band with intervals shown in gray and the higher frequency ("inner") split band with intervals shown in black. We use the terms outer and inner simply in the sense that frequency implies the particle number density and, loosely speaking, "height" in the solar atmosphere. The estimated interval for the inner band at $236.6 \mathrm{MHz}$ is uncertain due to overlapping herringbone fine structures. Thus we indicate our best estimate of the interval with a dashed (rather than solid) black line in 


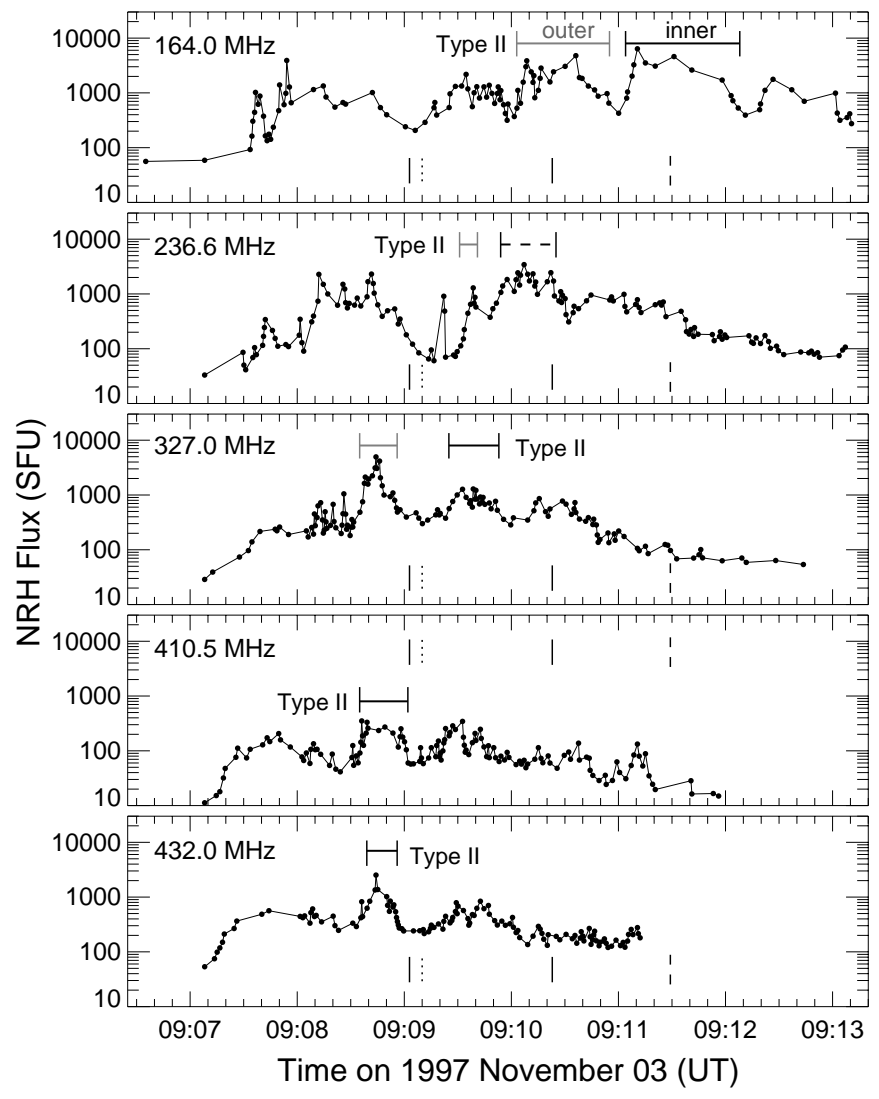

Fig. 10. Whole-Sun fluxes for each of the Nançay Radioheliograph frequencies (data courtesy K.-L. Klein) with estimated intervals for the outer (gray) and inner (black) split bands of the second harmonic mode emission of the type II burst. The tick marks represent the times of images as discussed in the main text.

Fig. 10. At the top of the plot for the $410.5 \mathrm{MHz}$ flux and at the bottom of the plots for the fluxes at the other frequencies we have drawn tick marks to indicate the times of (in sequential order) the SXT image shown in Fig. 3b (solid line), the $\mathrm{H} \alpha$ image when a Moreton wave is seen (dotted line), the SXT image shown in Fig. 3c (solid line), and the EIT $195 \AA$ image when an EIT wave is seen (dashed line).

\subsubsection{Type II source locations compared to the $\mathrm{H} \alpha$, EIT and SXT waves}

In Fig. 11 we present SXT, EIT and $\mathrm{H} \alpha$ difference images to show the features for the times indicated by the tick marks in Fig. 10. We also overplot contours for the closest $\mathrm{NRH}$ images to these times. In Fig. 11 contours in solid lines indicate inner band features of the second harmonic type II emission, while contours in broken (dotted or dashed) lines indicate outer band features. Furthermore, in each panel (but not between panels), the darker the contour lines the lower the frequency (and hence the lower the density) at the radio source sites. Note that in Figs. 11c,d a wider field of view is shown compared to Figs. 11a,b. The

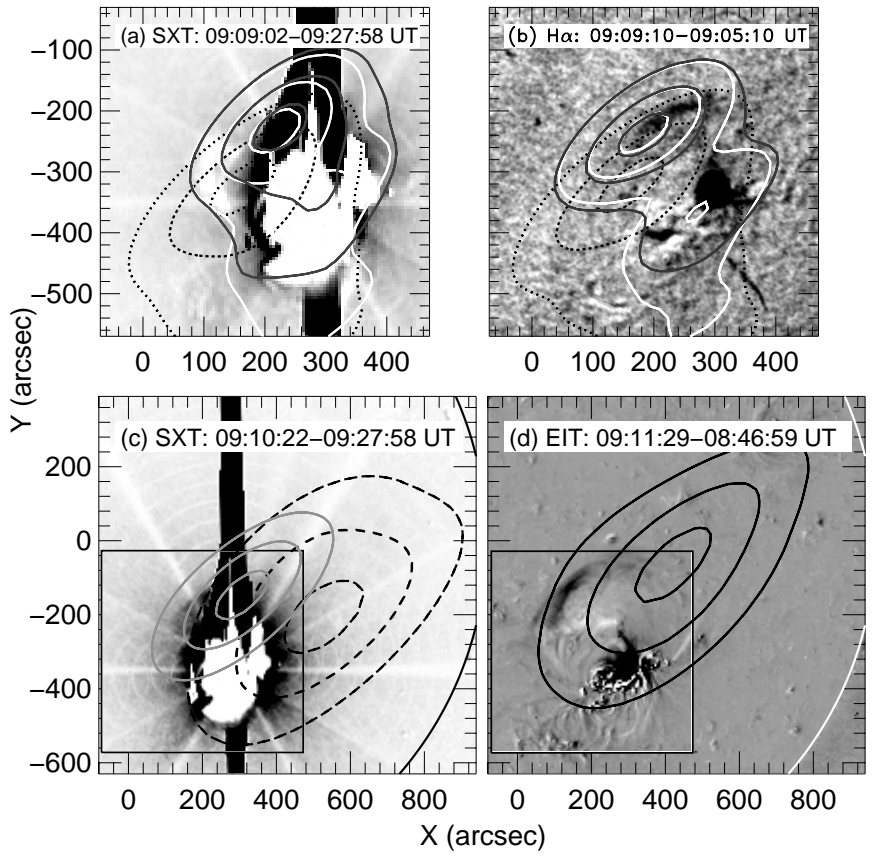

Fig. 11. Portions of SXT, $\mathrm{H} \alpha$, and EIT difference images with $30 \%, 60 \%$, and $90 \%$, contour levels for NRH images close to the first times indicated in each panel. The times and frequencies of the contours are described in the main text.

smaller field of view of Figs. 11a,b is shown by a boxed region in Figs. 11c,d.

From Fig. 10, the SXT image at 09:09:02.9 UT occurs after the inner band feature at $432 \mathrm{MHz}$, during the inner band feature observed at $410.5 \mathrm{MHz}$, and (probably) after the outer band feature at $327 \mathrm{MHz}$. In Fig. 11a we overplot contours for the $432 \mathrm{MHz}$ (solid white lines), $410.5 \mathrm{MHz}$ (solid dark gray lines), and $327 \mathrm{MHz}$ (dotted black lines) at 09:08:56.1 UT, 09:09:01.1 UT, and 09:09:01.1 UT, respectively. For the 432 and $410.5 \mathrm{MHz}$ source we show images slightly earlier than the time of the SXT image in order to show the location of the prior type II source more clearly, while for the $327 \mathrm{MHz}$ source we simply show the closest image to the time of the SXT image. We use a broken (dotted) line to indicate the $327 \mathrm{MHz}$ source as it is closest to the outer band feature. All contours show the main radio emission sources were to the northeast or east of the active region, but there was also an indication of a weaker source to the southwest over (or beyond) the active region. These NRH sources appear to indicate that the source of the type II emission is obscured by the saturation feature on the SXT CCD. This may explain why no clear SXT propagating disturbance was seen at this time.

From Fig. 10 the time of the $\mathrm{H} \alpha$ Moreton wave seen at 09:09:10 UT occurred after the inner band feature at $432 \mathrm{MHz}$ and $410.5 \mathrm{MHz}$, and approximately midway between the times of the outer and inner bands of the type II emission at $327 \mathrm{MHz}$ data. In Fig. 11b we use solid lines to indicate the $432 \mathrm{MHz}$ and $410.5 \mathrm{MHz}$ sources as these are closest to the inner band features and a dotted line to indicate the $327 \mathrm{MHz}$ source as it is slightly closer to the 
outer band feature. All sources show the main radio emission was to the northeast and east of the active region, with weaker emission over the active region. The main emission sources may be related to the prior clear type II emission. In Fig. 11b we overplot contours for the $432 \mathrm{MHz}$ (solid white lines), 410.5 MHz (solid dark gray lines), and $327 \mathrm{MHz}$ (dotted black lines) sources at 09:09:10.6 UT, 09:09:09.6 UT, and 09:09:10.1 UT, respectively. These are the closest NRH images to the time of the Moreton wave. The centers of the main sources at 432 and $410.5 \mathrm{MHz}$ appear to lie on part of the $\mathrm{H} \alpha$ Moreton wave at the time of that feature. We do not find a significant displacement between the centers of the main sources at $327 \mathrm{MHz}$ in Figs. 11a and 11b.

During the time of the SXT disturbance seen at 09:10:22.9 UT (as illustrated by the gray scale image in Fig. 11c) the inner band (herringbones) feature was observed at $236.6 \mathrm{MHz}$ and the outer band feature was observed at $164 \mathrm{MHz}$. In Fig. 11c we overplot contours for the $236.6 \mathrm{MHz}$ (solid light gray lines), and the $164 \mathrm{MHz}$ (dashed black lines) sources at 09:10:23.6 UT. The solar limb is indicated by a solid black line. The outer band $164 \mathrm{MHz}$ source lies 220 arcsec to the west-southwest of the inner band $236.6 \mathrm{MHz}$ source (which lies about 80 arcsec from the leading edge of the SXT feature). This suggests that the emission at 236.6 and $164 \mathrm{MHz}$ may occur at greater heights along the shock front where projection effects may be significant.

During the time of the EIT wave seen at 09:11:29.0 UT the inner band was observed at $164 \mathrm{MHz}$. In Fig. 11d we overplot contours for the $164 \mathrm{MHz}$ (solid black lines) at 09:11:3.1 UT. The solar limb is indicated by a solid white line. The center of the inner band $164 \mathrm{MHz}$ source appears to lie on part of the EIT wave. However, the $164 \mathrm{MHz}$ is expected to occur at a greater height than the $410.5 \mathrm{MHz}$ and $432 \mathrm{MHz}$ sources, and may be inclined with respect to those sources. Moreover, as will be seen later, the $164 \mathrm{MHz}$ source jumps around a lot. Thus the overlap we find here may simply be due to a fortuitous projection. We have ignored projection effects here as they are difficult to estimate reliably.

The center of the inner band $164 \mathrm{MHz}$ source in Fig. 11d was about 170 arcsec to the northeast of the center of the outer band $164 \mathrm{MHz}$ source in Fig. 11c. Aurass (1997) also reported widely separated sites for split band sources in another event. The separation of the inner band and outer band $164 \mathrm{MHz}$ sources may be due to a physical separation between inner and outer band features and the motion of the type II emitting regions with time. We also found that the center of the post-inner band $410.5 \mathrm{MHz}$ source in Fig. 11b was about 40 arcsec to the east of the inner band $410.5 \mathrm{MHz}$ source in Fig. 11a. A similar separation is found for the $432 \mathrm{MHz}$ sources seen in Figs. 11a and $11 \mathrm{~b}$.

Thus the NRH sources at the times of the $\mathrm{H} \alpha$ Moreton wave, the EIT wave and the SXT propagating disturbance suggest a close association with the type II burst source locations. There are also some indications of motion of

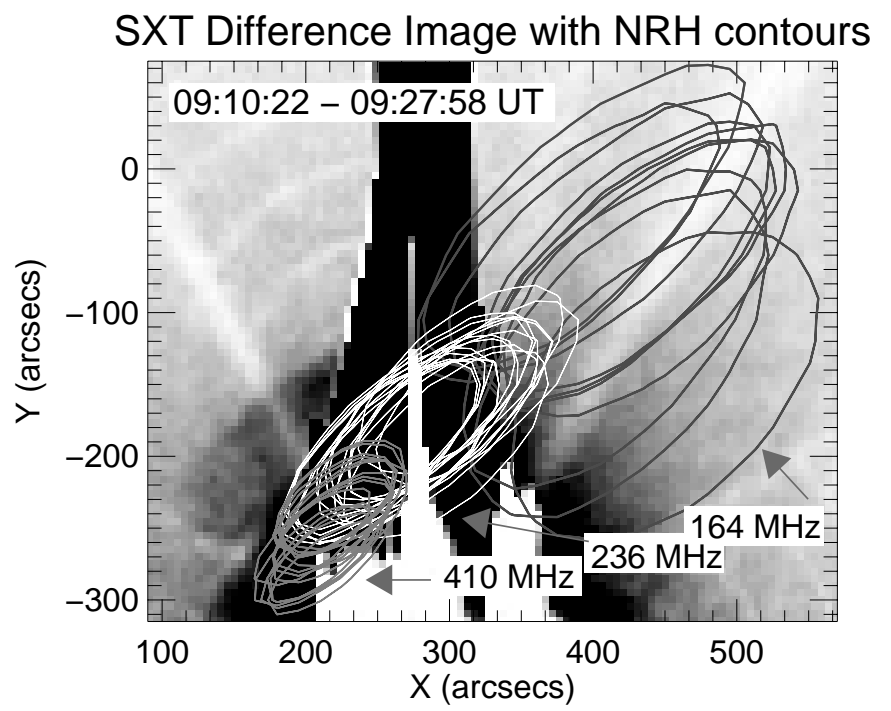

Fig. 12. The $90 \%$ contour levels for NRH images illustrating the motion of the inner band of the second harmonic mode of the type II radio sources.

the type II sources in the inferred direction of motion of the Moreton wave and the EIT wave, and the observed direction of motion of the SXT wave (i.e., the $410.5 \mathrm{MHz}$ and $432 \mathrm{MHz}$ sources in Figs. 11a,b and the $164 \mathrm{MHz}$ sources in Figs. 11c,d).

\subsubsection{General evolution of the type II source locations}

We now investigate the general evolution of the type II locations at NRH frequencies. As one illustration of our findings we show the $90 \%$ contour level for intervals of the inner band sources at 164, 236.6 and $410.5 \mathrm{MHz}$ in Fig. 12. The $164 \mathrm{MHz}$ contours are over the interval 09:11:0509:12:03 UT, while the $236.6 \mathrm{MHz}$ and $410.5 \mathrm{MHz}$ contours are over the intervals 09:09:55-09:10:23 UT and 09:08:36-09:09:01 UT, respectively. The $164 \mathrm{MHz}$ sources did not appear to lie on top of the SXT feature. The 236.6 MHz source appeared to be located behind the SXT feature. It is not clear whether the $410.5 \mathrm{MHz}$ source seen prior to the $236.6 \mathrm{MHz}$ source is located near the propagating soft X-ray disturbance as it is obscured by the saturation feature on the SXT CCD at the time.

While we do find that the positions of the type II sources jump around, nevertheless, we find some evidence supporting moving type II source locations. In Fig. 12 all sources show some evidence for either a northward motion or north-eastward motion roughly parallel to the direction of the propagating SXT disturbance.

To examine the motions of the NRH sources further we tracked the centroids of the type II radio burst sources. In order to define the centroids, we fitted a 2-D elliptical Gaussian function to each of the radio images. Although the shape of the fitted source was sometimes not a good match to the actual source shape, examination of the centroid locations of the fitted sources showed that these adequately describe the actual source centroids for 
single sources. We compared the fitted sources to the actual sources and eliminated those cases where the fits were not good (generally when there were two adjacent sources which occurred at times near the beginning or end of the type II intervals). However the $164 \mathrm{MHz}$ sources often showed closely adjacent double sources during the type II burst interval. Because there are many of these sources and they occur throughout the interval we, nevertheless, retain the fits to these sources but bear in mind that there is greater uncertainty in the $164 \mathrm{MHz}$ source locations compared to other frequencies using the 2-D elliptical Gaussian fitting technique.

Figure $13 \mathrm{a}$ shows the $x$ - and $y$-coordinates (in $\mathrm{NRH}$ (15 arcsec) pixels) of the centroid fits to the NRH sources at different frequencies during the intervals of the type II burst. Each symbol represents a different NRH frequency (as indicated in the figure caption). Because the sources jump around, systematic motions are not obvious from this plot. The two plots on the right-hand side show separate plots for the $x$ - and $y$-coordinates with time. These indicate possible systematic motions of the centroids of the radio source locations. The clearest examples are the $y$-coordinates for the 236.6 and $410.5 \mathrm{MHz}$ inner band features. Moreover, the displacement between the $y$-coordinates of the mean values of the centroids of the groups of outer and inner band $327 \mathrm{MHz}$ points (indicated with a dotted line in Fig. 13c) shows a slope similar to that seen for the sets of individual points along the 236.6 and $410.5 \mathrm{MHz}$ inner band features. A steeper slope is seen for the line joining the mean value of the centroids of the groups of outer and inner band 236.6 MHz sources. The $164 \mathrm{MHz}$ inner band locations indicate a possible trend of a drift to larger $y$-coordinates with time. Thus the plot of the $y$-coordinates indicate a generally northward motion of the inner band features, and in some cases a northward motion comparing the relative displacement of the mean positions of the groups of outer and inner band features. The $x$-coordinates do not show consistent systematic motions. The set of inner band $164 \mathrm{MHz}$ centroid positions indicate possible eastward motion (i.e., to smaller $x$-coordinates), as does the general trend comparing the groups of outer and inner band points at $164 \mathrm{MHz}$. The displacement between the $x$-coordinates of the mean values of the centroids of the groups of outer and inner band $236.6 \mathrm{MHz}$ points indicates possible eastward motion. A similar line joining the mean values of the groups of outer and inner band $327 \mathrm{MHz}$ points indicates either no significant east-west motions or possibly slight westward motions. Similarly for the fit to the set of inner band 410.5 MHz sources.

Linear fits to the $y$-coordinates for the 410.5, 236.6 and $164 \mathrm{MHz}$ inner band sources yield speeds along that coordinate of 1082, 1319 and $538 \mathrm{~km} \mathrm{~s}^{-1}$, respectively. Linear fits to the $x$-coordinates for the 410.5 and $164 \mathrm{MHz}$ inner band sources yield speeds along that coordinate of 435 and $-741 \mathrm{~km} \mathrm{~s}^{-1}$, respectively. Thus the speeds for the 410.5 and $164 \mathrm{MHz}$ inner band sources are 1166 and $916 \mathrm{~km} \mathrm{~s}^{-1}$, respectively. The "speed" implied by comparing the mean positions of the groups of outer and inner band $327 \mathrm{MHz}$ features is $933 \mathrm{~km} \mathrm{~s}^{-1}$. It should be noted that these NRH speeds do not measure the speed of the shock wave causing the type II emission. The location emitting type II bursts at a given NRH frequency is the intersection of the shock with a particular particle density level (i.e., height) in the solar atmosphere. Let us suppose that the shock wave propagates outward as an expanding spherical shell in a plane-parallel atmosphere. Then the location emitting type II bursts at a given NRH frequency would be the intersection of the expanding spherical shell (representing the shock wave) with a horizontal plane surface (representing a fixed particle density level). Clearly the location of this intersect will propagate faster than the shock wave initially, but will asymptotically approach the shock wave speed at later times.

In summary we find that the $\mathrm{NRH}$ data show the type II radio bursts were located close to the soft X-ray propagating disturbance. They also show indications of motions generally consistent with the soft X-ray motions.

\section{Conclusions}

In this paper we have presented observations of a propagating disturbance seen in emission in soft X-ray images of the solar corona after the onset of a flare which began at about 09:04 UT on 1997 November 03. The propagating feature was seen clearly in two SXT images. It had a sharply defined leading edge with a similar level of enhanced emission behind it. Using a ray-tracing technique for points along each of the leading edges, and the reasonable assumption that these features are closely related to each other, we can determine the average speed of propagation between them. We find that the disturbance traveled with a speed of about $546 \pm 21 \mathrm{~km} \mathrm{~s}^{-1}$ over the interval 09:10:22-09:12:30 UT.

This event was also associated with an $\mathrm{H} \alpha$ Moreton wave and an EIT wave. We note that the $\mathrm{H} \alpha$ Moreton wave, EIT wave, and propagating soft X-ray disturbance all occurred during the interval when a strong type II burst was seen by the AIP Radio Spectrograph. Using a raytracing technique we extrapolated the leading edges of the SXT propagating disturbance to the times of the Moreton wave and the EIT wave. The extrapolations closely overlap the actual observed locations of the Moreton and EIT waves. This indicates not only that the propagating soft X-ray disturbance is closely associated with the $\mathrm{H} \alpha$ Moreton wave and the EIT wave, but suggests that the latter two waves are also related to each other. Values for the speed of the shock wave (assumed to give rise to the type II radio burst), estimated from radio spectrograms, are strongly dependent on the particle density model of the solar atmosphere used. Nevertheless, we find that the speed inferred using the Mann et al. (1999) model implies a speed of $\sim 560 \mathrm{~km} \mathrm{~s}^{-1}$, which is comparable to the speed determined for the propagating soft X-ray disturbance.

Using Nançay Radioheliograph data we directly determine the source locations of the type II radio burst. 

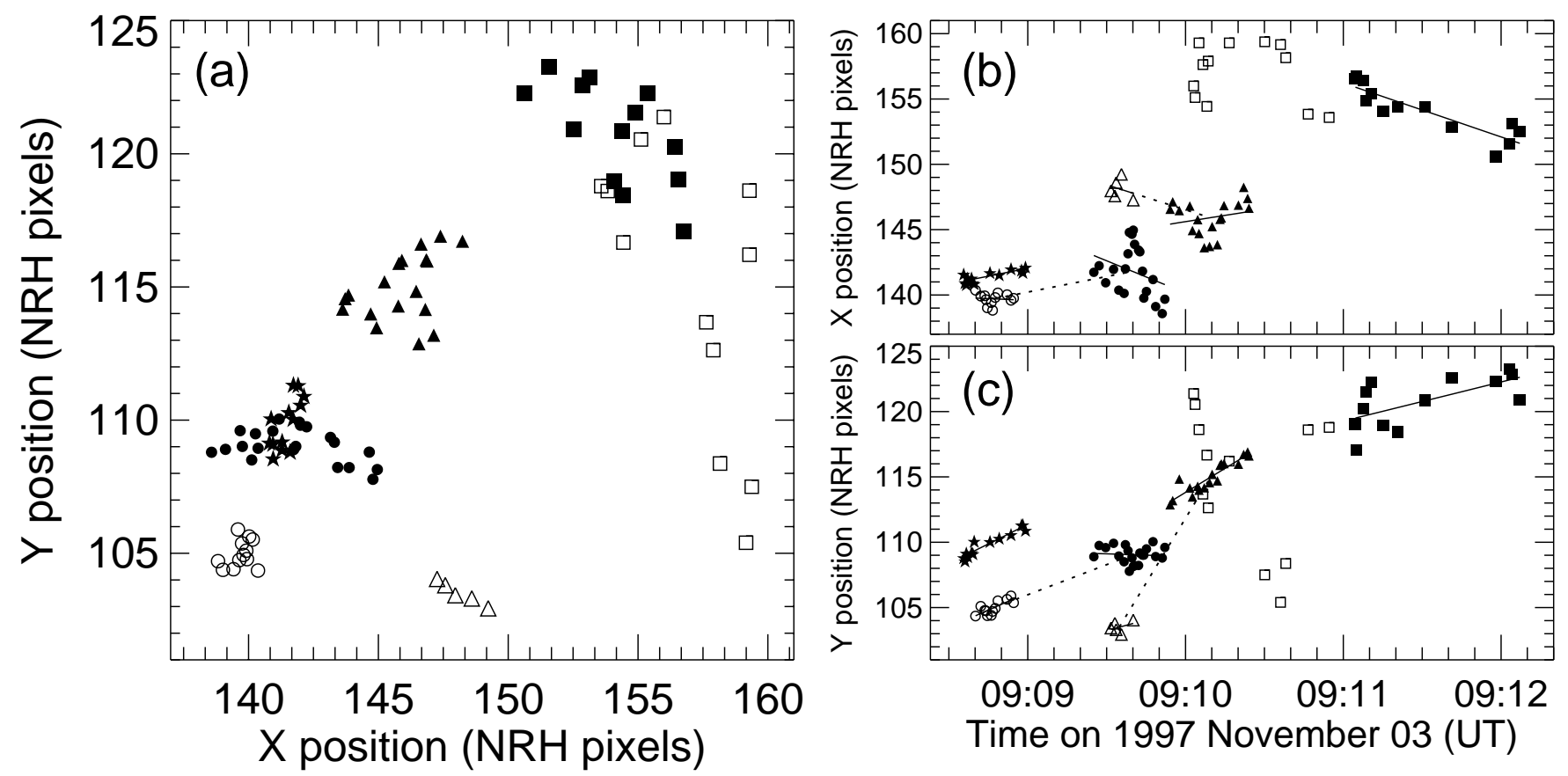

Fig. 13. a) The $x$ - and $y$-coordinates (in NRH pixel units) of the centroids of 2-D elliptical Gaussian function fits to the type II radio sources, $\mathbf{b}$ ) and $\mathbf{c}$ ) show the $x$ - and $y$ - coordinates for these centroids as a function of time. Open symbols indicate outer band features while the filled symbols indicate inner band features. The centroid locations of the sources at 164, 236.6, 327, and $410.5 \mathrm{MHz}$ are shown with square, triangle, circle and star symbols, respectively. Solid lines indicate the best linear fits to the sets of some of the inner or outer band points. Dotted lines join the mean values of outer and inner band features at a given frequency.

The NRH data show the type II radio burst was located in the vicinity of the $\mathrm{H} \alpha$ Moreton wave, the EIT wave and the SXT propagating disturbances at the times of these features (given the relatively poor spatial resolution at radio wavelengths). The NRH sources also show motions generally consistent with the motions for the propagating soft X-ray disturbance. This indicates a possible relation between the SXT propagating disturbance and the source locations of the type II burst.

It has not yet been firmly established how to distinguish whether features observed in the SXT data are loops, waves, or ejecta. For example, moving features associated with flares on 8 and 9 May 1998 were attributed to shock waves by Khan \& Hudson (2000) but to material (loop or plasmoid) ejection by Nitta \& Akiyama (1999). We note that the propagating soft X-ray feature seen initially in the event discussed here has a sharp leading edge and does not appear obviously loop-like (the soft X-ray features do not extend to clearly anchored footpoints). The close correspondence temporally and spatially (in shape and location) with the Moreton and EIT waves appears to support the suggestion that the early soft X-ray features are closely related to the shock wave itself. Our results lead us to conclude that the Yohkoh SXT is able to observe the coronal counterpart of Moreton waves, analogous to EIT $195 \AA$ waves. In other words, we suggest that the early SXT propagating disturbance is the first confirmed direct observation of a coronal shock wave seen in soft X-rays.
Acknowledgements. We are grateful to Dr. H. S. Hudson for useful discussions and comments. The Yohkoh mission is a project of the Institute of Space and Astronautical Science (ISAS) of Japan. It is financially supported by ISAS, NASA, and the Particle Physics and Astronomy Research Council (PPARC) of the UK. The Nançay Radio Observatory is funded by the French Ministry of Education, the Centre National de la Recherche Scientifique (CNRS), and the Région Centre. We are grateful to Dr. K.-L. Klein for generously providing the Nançay Radioheliograph data. The EIT data used here are courtesy of the SOHO EIT Consortium. SOHO is a joint ESA and NASA project. The $\mathrm{H} \alpha$ data used here are courtesy of Kanzelhöhe Solar Observatory, Treffen, Austria. We are grateful to W. Otruba and A. Warmuth for providing that data. The GOES data are courtesy of the World Data Center A for Solar-Terrestrial Physics, NGDC, NOAA E/GC2, 325 Broadway, Boulder, Colorado 80303, USA. J.I.K. received support from PPARC. H.A. is grateful to the Japan Society for the Promotion of Science, and to the Deutscher Akademischer Austauschdienst, for generously funding his working stays at ISAS.

\section{References}

Aurass, H. 1997, in Coronal Physics from Radio and Space Observations, ed. G. Trottet (Berlin: Springer-Verlag), Lect. Notes Phys., 483, 135

Delaboudinière, J.-P., Artzner, G. E., Brunaud, J., et al. 1995, Sol. Phys., 162, 291

Delannée, C. 2000, ApJ, 545, 512

Delannée, C., \& Aulanier, G. 1999, Sol. Phys., 190, 107 
Delannée, C., Delaboudinière, J.-P., \& Lamy, P. 2000a, Adv. Space Res., 26, 67

Delannée, C., Delaboudinière, J.-P., \& Lamy, P. 2000b, A\&A, 355,725

Ding, M. D., Watanabe, T., Shibata, K., et al. 1996, ApJ, 458, 391

Domingo, V., Fleck, B., \& Poland, A. I. 1995, Sol. Phys., 162, 1

Gopalswamy, N., Kaiser, M. L., Sato, J., \& Pick, M. 2000, in High Energy Solar Physics -Anticipating HESSI, ed. R. Ramaty, \& N. Mandzhavidze (San Francisco: Astronomical Society of the Pacific), ASP Conf. Ser., 206, 351

Harvey, K. L., Martin, S. F., \& Riddle, A. C. 1974, Sol. Phys., 36,151

Hudson, H. S., \& Karlický, M. 2000, in High Energy Solar Physics -Anticipating HESSI, ed. R. Ramaty, \& N. Mandzhavidze (San Francisco: Astronomical Society of the Pacific), ASP Conf. Ser., 206, 268

Kai, K. 1969, Sol. Phys., 10, 460

Kerdraon, A., \& Delouis, J.-M. 1997, in Coronal Physics from Radio and Space Observations, ed. G. Trottet (Berlin: Springer-Verlag), Lect. Notes Phys., 483, 192

Karlický, M., Jiřička, K., Kepka, O., Křivský, L., \& Tlamicha, A. 1982, Bull. Astron. Inst. Czechoslovakia, 33, 72

Khan, J. I., \& Hudson, H. S. 2000, GRL, 27, 1083

Klassen, A., Aurass, H., Klein, K.-L., Hofmann, A., \& Mann, G. 1999, A\&A, 343, 287

Klassen, A., Aurass, H., Mann, G., \& Thompson, B. J. 2000, A\&AS, 141, 357

Klassen, A., Aurass, H., \& Mann, G. 2001, A\&A, 370, L41

Klein, K.-L., Khan, J. I., Vilmer, N., Delouis, J.-M., \& Aurass, H. 1999, A\&A, 346, L53

Leblanc, Y., Dulk, G. A., Vourlidas, A., \& Bougeret, J.-L. 2000, GRL, 18, 18225

Mann, G. 1995, in Coronal Magnetic Energy Releases, ed. A. O. Benz, \& A. Krüger (Berlin: Springer-Verlag), Lect. Notes Phys., 44, 183

Mann, G., Aurass, H., Voigt, W., \& Paschke, J. 1992, in Coronal Streamers, Coronal Loops and Coronal and Solar Wind Composition (Noordwijk: ESA), ESA SP-348, 129

Mann, G., Jansen, F., MacDowall, R. J., Kaiser, M. L., \& Stone, R. G. 1999, A\&A, 348, 614

Mariska, J. T. 1994, ApJ, 434, 756

Maxwell, A., \& Dryer, M. 1982, Space Sci. Rev., 32, 11

Maxwell, A., Dryer, M., \& McIntosh, P. 1985, Sol. Phys., 97, 401

Meyer, F. 1968, in Structure and Development of Solar Active Regions, ed. K. O. Kiepenheuer (Dordrecht: D. Reidel Publ. Co.), IAU Symp., 35, 485

Moreton, G. E. 1960, AJ, 65, 494
Moreton, G. E. 1961, Sky and Telescope, 21, 145

Moreton, G. E. 1964, AJ, 69, 145

Moreton, G. E., \& Ramsey, H. R. 1960, PASP, 72, 357

Moses, D., Clette, F., Delaboudinière, J.-P., et al. 1997, Sol. Phys., 175, 571

Nelson, G. J., \& Melrose, D. B. in Solar Radiophysics, ed. D. J. McLean, \& N. R. Labrum (Cambridge: Cambridge University Press), 333

Newkirk, G., Jr. 1961, ApJ, 133, 983

Nitta, N., \& Akiyama, S. 1999, ApJ, 525, L57

Ogawara, Y., Takano, T., Kato, T., et al. 1991, Sol. Phys., 136,1

Pohjolainen, S., Maia, D., Pick, M., et al. ApJ, 556, 421

Ramsey, H. R., \& Smith, S. F. 1966, AJ, 71, 197

Roberts, J. A. 1959, Aust. J. Phys., 12, 327

Robinson, R. D. 1985, Sol. Phys., 95, 343

Smith, E. v. P. 1968, in Mass Motions, Solar Flares and Related Phenomena, ed. Y. Öhman (Stockholm: Almquist \& Wiksell), Nobel Symp., 9, 137

Smith, S. F., \& Harvey, K. L. 1971, in Physics of the Solar Corona, ed. C. J. Macris (Dordrecht: D. Reidel Publ. Co.), 156

Thompson, B. J., Plunkett, S. P., Gurman, J. B., et al. 1998, GRL, 25, 2461

Thompson, B. J., Gurman, J. B., Neupert, W. M., et al. 1999, ApJ, 517, L151

Thompson, B. J., Reynolds, B., Aurass, H., et al. 2000, Sol. Phys., 193, 161

Tsuneta, S., Acton, L. W., Bruner, M. E., et al. 1991, Sol. Phys., 136, 37

Uchida, Y. 1960, PASJ, 12, 376

Uchida, Y. 1968, Sol. Phys., 4, 30

Uchida, Y. 1970, PASJ, 22, 341

Uchida, Y. 1974, Sol. Phys., 39, 431

Uchida, Y., Altschuler, M. D., \& Newkirk, G., Jr. 1974, Sol. Phys., 39, 431

Vršnak, B., \& Lulić, S. 2000, Sol. Phys., 196, 181

Warmuth, A., Vršnak, B., Aurass, H., \& Hanslmeier, A. 2001, ApJ, 560, L105

Wild, J. P. 1950, Aust. J. Sci. Res. A, 3, 399

Wild, J. P., Murray, J. D., \& Rowe, W. C. 1954, Austr. J. Phys., 7, 439

Wild, J. P. 1985, in Solar Radiophysics, ed. D. J. McLean, \& N. R. Labrum (Cambridge: Cambridge University Press), 3

Wild, J. P., \& McCready, L. L. 1950, Aust. J. Sci. Res. A, 3, 387

Wild, J. P., \& Smerd, A. F. 1972, ARA\&A, 10, 159

Wills-Davey, M. J., \& Thompson, B. J. 1999, Sol. Phys., 190, 467 